\title{
Complex dynamics in nanoscale phase separated supercooled liquids
}

\author{
S. Cazzato, ${ }^{1}$ A. Chrissanthopoulos $\odot,{ }^{2}$ M. Micoulaut, ${ }^{3,}{ }^{*}$ T. Scopigno,,${ }^{4,5}$ and S. N. Yannopoulos $\odot^{6}$ \\ ${ }^{1}$ Research Center SFT, INFM, CNR, Università di Roma "La Sapienza," 00185 Roma, Italy \\ ${ }^{2}$ Department of Chemistry, National and Kapodistrian University of Athens, 15784 Zografou, Athens, Greece \\ ${ }^{3}$ Sorbonne Université, Laboratoire de Physique Théorique de la Matière Condensée, CNRS UMR 7600, 4 Place Jussieu, \\ 75252 Paris Cedex 05, France \\ ${ }^{4}$ Dipartimento di Fisica, Università di Roma “La Sapienza”, I-00185 Rome, Italy \\ ${ }^{5}$ Istituto Italiano di Tecnologia, Center for Life Nano Science @ Sapienza, Roma, I-00161, Italy \\ ${ }^{6}$ Foundation for Research and Technology Hellas Institute of Chemical Engineering Sciences (FORTH/ICE-HT), \\ P.O. Box 1414, 26504 Rio-Patras, Greece
}

(Received 5 May 2020; revised 12 June 2020; accepted 15 June 2020; published 7 July 2020)

\begin{abstract}
The relaxation properties of supercooled $\mathrm{As}_{x} \mathrm{~S}_{100-x}$ liquids are investigated using a combination of infrared photon correlation spectroscopy and topological constraint theory. Results reveal two channels of relaxation for sulfur-rich compositions that manifest by an unusual profile in the density-density autocorrelation function involving two typical timescales. This indicates a reduced temperature-dependent dynamics for one of the channels associated with a sulfur-rich segregated nanoscale phase that furthermore displays a low liquid fragility. Conversely, the dynamics of the emerging cross-linked As-S network is associated with a growth of the glass transition temperature with As content. These results can be quantitatively understood from topological constraint theory applied to a phase separated network for which a dedicated constraint enumeration must be achieved. The vanishing of this peculiar behavior occurs close to the reported isostatic reversibility window observed at the glass transition.
\end{abstract}

DOI: 10.1103/PhysRevResearch.2.032007

The way viscosity $\eta$ or relaxation time $\tau_{\alpha}$ increases with decreasing temperature in supercooled liquids continues to be an object of intense research activities, although the salient features of glassy relaxation are rather well documented [1]. These manifest in a dramatic increase of $\eta$ and $\tau_{\alpha}$ within small temperature changes, and a glass transition temperature $T_{g}$ can be defined once $\eta=10^{12} \mathrm{Pas}$ or $\tau_{\alpha}=10^{2} \mathrm{~s}$. The onset of glassy dynamics for $T>T_{g}$ can be tracked from density-density correlation functions in Fourier space encoded in an intensity autocorrelation or intermediate scattering function $F_{s}(k, t)$ [or $g_{2}(k, t)$ ] that can be measured from various experimental probes, including the more recent $\mathrm{x}$-ray photon correlation spectroscopy technique [2-4]. For a variety of materials and experimental conditions, the obtained $g_{2}(k, t)$ has a complex but standard profile, i.e., it continuously decays to $g_{2}(k, t) \rightarrow 0$ for long times but reveals multiple relaxation steps $[5,6]$ as the temperature decreases, from short-time relaxation ( $\beta$ relaxation) to a final and longer timescale relaxation ( $\alpha$ relaxation) that can be appropriately fitted using a stretched Kohlrausch-Williams-Watts (KWW) exponential $\exp \left[-\left(t / \tau_{\alpha}\right)^{\beta}\right]$ which departs from the usual Debye decay at high temperature. Here both $\tau_{\alpha}$ and $\beta$ depend on temperature and composition for a fixed momentum transfer $k$.

\footnotetext{
*Corresponding author: mmi@lptmc.jussieu.fr

Published by the American Physical Society under the terms of the Creative Commons Attribution 4.0 International license. Further distribution of this work must maintain attribution to the author(s) and the published article's title, journal citation, and DOI.
}

For certain supercooled liquids such as chalcogenides, the $g_{2}(k, t)$ function and the underlying relaxation phenomena cannot be studied in the usual way. Such systems are also known to undergo a flexible to rigid transition [7]. In this Rapid Communication we take advantage of infrared photon correlation spectroscopy (IRPCS) which we introduce precisely to address the relaxation dynamics of sulfide glassforming liquids. We show that these display an unusual decay for the relaxation function, suggesting a more complicated relaxation dynamics. For select sulfur-rich compositions in binary As-S liquids, $g_{2}(k, t)\left[g_{2}(t)\right.$ hereafter] displays indeed two channels of relaxation with a rapid relaxation rate $\tau_{\alpha}^{1}$ driving the onset of the glass transition for an As-rich atomic structure, whereas a slower relaxation $\tau_{\alpha}^{2}$ appears to be linked with a sulfur-rich nanophase structure that is decoupled from the network and reminiscent of the $T_{\lambda}$ transition that is peculiar to elemental sulfur [8]. A modified Mauro-Gupta model [9] permits one to not only predict the $T_{g}$ variation with As content, but also decode the effect of the nanoscale phase separation on the observed relaxation properties. Complex relaxation functions have been investigated for phase separated mixtures with a loose network structure (e.g., hard spheres [10]) or high-molecular-weight molecules in solvents [11]. For the latter, slow and fast modes are assigned to pure translational diffusion and intramolecular motion, respectively. In the present case, given the change in chemical composition, the observed complex function $g_{2}(t)$ in S-rich melts appears rather to be deeply influenced by structural aspects at the nanoscale, As cross-linking, and phase separation, whereas the corresponding interactions can be simply modeled using topological constraints. The observation appears therefore to 
have some unique and nontrivial character regarding network glass-forming liquids.

Glasses $\left(\mathrm{As}_{x} \mathrm{~S}_{100-x}\right)$ with compositions $x=10,20,30$, and 40, were prepared from high-purity elements $(99.9999 \%$, Alfa Aesar). Appropriate amounts of the elements, of a total mass of $\simeq 1.5 \mathrm{~g}$, were loaded in silica tubes. Due to the high propensity of As to oxidation, all operations took place in an inert (Ar) atmosphere glove box with water and oxygen content less than 2 ppm. Before being loaded with the chemicals, silica tubes were thoroughly cleaned by dilute hydrofluoric acid and rinsed several times with triple distilled water. The tubes were degassed and baked by an oxygen-butane flame at $1100^{\circ} \mathrm{C}$ in order to enhance their optical quality. The ampoules loaded with the chemicals were sealed under vacuum $\left(10^{-5}\right.$ Torr $)$ and were placed in a furnace in which the materials were heated up to a temperature at about $100^{\circ} \mathrm{C}-200^{\circ} \mathrm{C}$ above the liquidus point of each alloy. The melts were kept at high temperature for at least $48 \mathrm{~h}$ with periodic shaking in order to ensure their homogenization, before being quenched in water.

Being low band-gap semiconductors, chalcogenide glasses are sensitive to visible light, which induces a number of photostructural effects [12]. The situation becomes more complex in the supercooled regime where the band gap is even narrower and light absorption is significant. The use of infrared radiation allows one to overcome photoinduced and absorption effects at visible wavelengths, and this technique was introduced earlier by Scopigno et al. [8]. During the present IRPCS investigation the setup operated at a fixed value of the scattering angle $\left(\theta \simeq 90^{\circ}\right)$, although other angles were considered but the relaxational dynamics was found to be angle independent. Light scattering data were recorded in the supercooled liquid regime and the system was kept at each temperature at least $20 \mathrm{~min}$ for equilibration, before each acquisition. Several acquisition runs were performed for each sample in order to check reproducibility.

The main outcome from IRPCS measurements is the scattered field correlation function, directly related to the densitydensity time correlation function for an isotropic medium in polarized scattering geometry [8]. A representative set of the measured correlation functions is given in Fig. 1 for two select compositions ( $\mathrm{As}_{10} \mathrm{~S}_{90}$ and $\mathrm{As}_{40} \mathrm{~S}_{60}$, open circles), together with reduced homodyne correlation functions of the form $g_{2}(t)$ (red solid lines),

$$
g_{2}(t)=\sum_{i=1}^{N} A_{i} \exp \left[-\left(t / \tau_{\alpha}^{i}\right)^{\beta_{i}}\right],
$$

containing more than one stretched exponential KWW decay channel characterized by $A_{i}, \tau_{\alpha}^{i}$, and $\beta_{i}$. As can be seen, such a model with KWW functions indicates that $N=1$ is enough for $x=30$ and 40 , whereas the unusual behavior for $x \leqslant 20 \%$ needs $N>1$ to be reproduced correctly and this induces the presence of two relaxation times $\tau_{\alpha}^{1}$ and $\tau_{\alpha}^{2}$ corresponding to shorter and longer timescales, respectively.

Figure 2(a) illustrates the relaxation times $\tau_{\alpha}^{1}$ and $\tau_{\alpha}^{2}$ obtained from the fit of the normalized autocorrelation functions. The complex decay of the latter for As compositions less than $20 \%$ leads to relaxation channels whose characteristic times differ by more than two orders of magnitude. For example, for $10^{3} / T=1.8(555 \mathrm{~K})$ one finds $\log _{10} \tau_{\alpha}^{1}=-4.06$ and

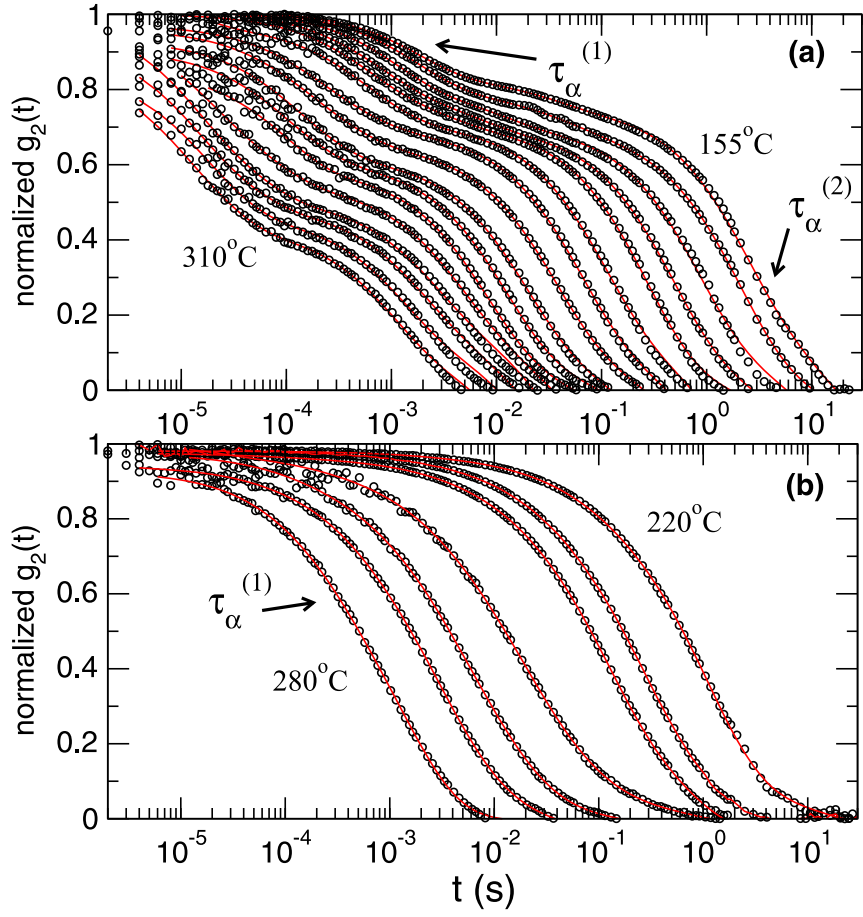

FIG. 1. Normalized intensity autocorrelation functions $g_{2}(t)$ from supercooled $\mathrm{As}_{x} \mathrm{~S}_{100-x}$ (circles) for two select compositions: (a) $x=10 \%$ and (b) $x=40 \%$. The solid lines represent the model function (1). For each composition the higher and lower experimentally accessed temperatures are reported. Temperature steps are each $10^{\circ} \mathrm{C}$ up to the maximum temperature.

$\log _{10} \tau_{\alpha}^{2}=-1.52$ for the $\mathrm{As}_{20} \mathrm{~S}_{80}$ liquid; a similar difference holds for the other S-rich composition. One should note that the difference between both $\tau_{\alpha}^{k}$ is maintained up to high temperature (e.g., $310^{\circ} \mathrm{C}$ for $\mathrm{As}_{10} \mathrm{~S}_{90}$ ), so the channels cannot be associated with a separate primary $\alpha$-relaxation process and a secondary $\beta$-relaxation process as both are known to coalesce at high temperature. Here different noncrossing timescales with a "parallel" $T$ dependence are acknowledged instead so that their origin can be regarded as different. The temperature behavior of $\tau_{\alpha}^{k}$ can be fitted using a Vogel-Fulcher-Tamman (VFT) or a Mauro-Yue-Ellison-Gupta-Allan (MYEGA) [15] fit, the latter being given by

$$
\begin{aligned}
\log _{10} \tau_{\alpha}(T)= & \log _{10} \tau_{\infty}+\left(2-\log _{10} \tau_{\infty}\right) \frac{T_{g}}{T} \\
& \times \exp \left[\left(\frac{m}{2-\log _{10} \tau_{\infty}}-1\right)\left(T_{g} / T-1\right)\right],
\end{aligned}
$$

where $\tau_{\infty}, T_{g}$, and $m$ are the relaxation time at infinite temperature, the glass transition temperature, and the fragility, respectively. Note that the difference between both fits is barely visible [Fig. 2(a)] as both fits to the data lead to a correlation coefficient greater than 0.99 . Figure 2(b) represents the fragilities obtained from both fits (VFT and MYEGA) of the IRPCS data. Results indicate a fragility for the slow process that is nearly constant at about $m=m_{2} \simeq 15$ and which arises from the long-time relaxation behavior characterized by $\tau_{\alpha}^{2}$. Conversely, the fragility of the faster process increases with 

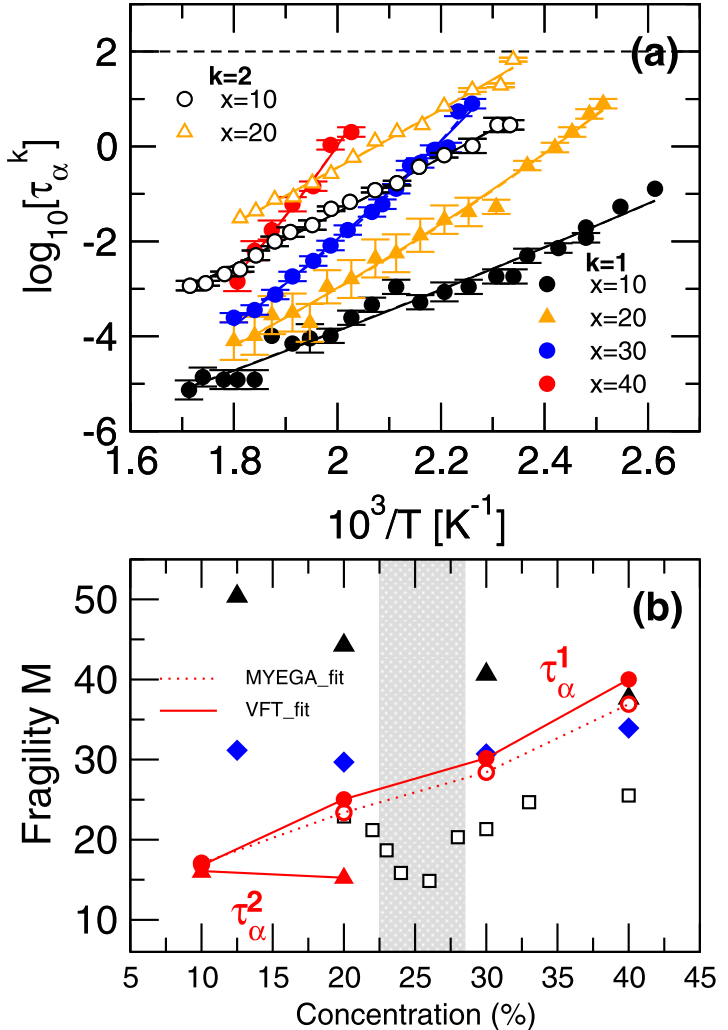

FIG. 2. (a) Relaxation time $\tau_{\alpha}^{k}[k=1$ (closed symbols) and $k=$ 2 (open symbols)] as a function of inverse temperature for supercooled As-S melts. The VFT and MYEGA fits (not distinguishable) are represented by solid and dashed lines, respectively. (b) Fragility evolution $m$ as a function of As content, obtained from MYEGA and VFT fits. Open squares are results from calorimetry [13], closed triangles are viscosity data [14], and blue diamonds are obtained by extrapolating $\eta / G$ to $100 \mathrm{~s}$. The gray zone indicates the reversibility window compositions [13].

As content to reach a value of about $m_{1} \simeq 38$ for $\mathrm{As}_{2} \mathrm{~S}_{3}$, compatible with viscosity-based measurements. The data appear to be somewhat different from fragilities determined from calorimetric measurements and associated with enthalpic relaxation at the glass transition which furthermore display a minimum for so-called reversibility window compositions underscoring a topological intermediate phase [16] [gray zone in Fig. 2(b) [13]].

Our interpretation is as follows. The structure of S-rich glasses is locally phase separated with regions I where the structure is dominated by an As-S network backbone and regions II where $\mathrm{S}_{8}$ rings and $\mathrm{S}_{n}$ chains dominate. As a result, two glass transition temperatures are observed from calorimetry [7,17], the endothermic one at $\simeq 150{ }^{\circ} \mathrm{C}$ being identified as the signature of the $T_{\lambda}$ transition. Such $\mathrm{S}_{8}$ rings are also visible from the Raman spectra of glasses at specific wave numbers, and corresponding peaks vanish for $x>24 \%$ As [13], i.e., there are no more rings left in glasses with a larger As content. For $T>T_{\lambda}$, the $\mathrm{S}_{8}$ rings polymerize into $\mathrm{S}_{n}$ chains [7], but the large viscosity in the $T_{\lambda}$ region prevents a complete remixing of both phases. As a result, the structure of S-rich melts continues to be locally phase separated with two regions having different timescales $\tau_{\alpha}^{1}$ and $\tau_{\alpha}^{2}$ arising from

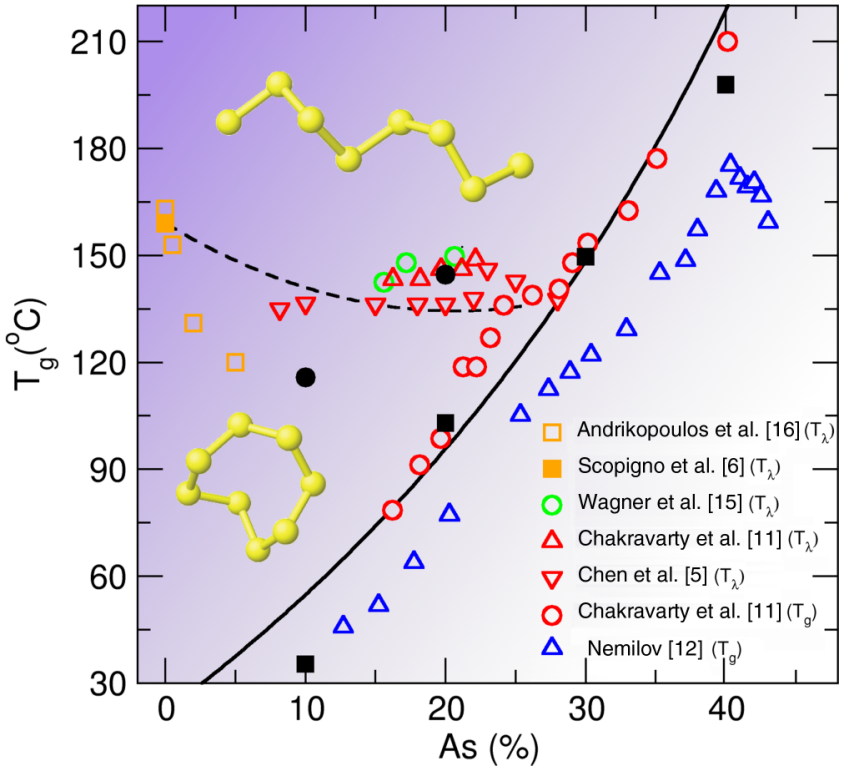

FIG. 3. Typical temperature $T_{g}^{(2)}$ and glass transition temperature $T_{g}^{(1)}$ obtained from the fit (MYEGA) of the IRPCS data (black closed squares from $\tau_{\alpha}^{1}$ and black closed circles from $\tau_{\alpha}^{2}$ ), compared to $T_{g}$ data from Nemilov (viscosity [14]) and Chakravarty et al. (calorimetry [13]). The solid curve is a prediction of $T_{g}(x)$ from TCT with $T_{g}(0)=295 \mathrm{~K}$ [Eq. (3)]. Also represented are the $T_{\lambda}$ data of Wagner et al. [17], Chen et al. [7], Chakravarty et al. [13], and Andrikopoulos et al. [18]. The $T_{\lambda}$ of pure sulfur is indicated $[8,18]$. The dashed curve serves only as a guide.

structure-related relaxation processes (diffusion, reorientation, etc.), the former still being dominated by the As-S network.

In fact, the fits [e.g., Eq. (2)] of Fig. 2(a) also permit access to the glass transition temperatures defined by $\log _{10} \tau_{\alpha}^{k}=2$ and the glass transition temperature of the $k=1$ channel $\left(T_{g}^{(1)}\right)$ is actually clearly comparable to the one determined from calorimetry for $\mathrm{As}_{x} \mathrm{~S}_{100-x}$ glasses and therefore linked with phase I containing mostly the network of $\mathrm{AsSe}_{3 / 2}$ pyramidal units whose growing presence induces an overall increase of the network connectivity that is reflected in the increase of $T_{g}$ with As content (Fig. 3). We do find indeed that when represented as a function of composition, $T_{g}^{(1)}$ (closed squares) behaves very similarly to the calorimetric $T_{g}$, if not exactly, which indicates indeed that the short-time relaxation is the one driven by the cross-linking As in the network structure. Conversely, the dynamics associated with $\tau_{\alpha}^{2}$ for $10 \%$ and $20 \%$ is linked with the equilibrium between monomers $\left(\mathrm{S}_{8}\right)$ and polymers $\left(\mathrm{S}_{n}\right)$. For the latter, a corresponding typical temperature $T_{g}^{(2)}$ fulfilling $\tau_{\alpha}^{2}\left(T_{g}^{(2)}\right)=10^{2} \mathrm{~s}$ nearly coincides with $T_{\lambda}$ [7,17] (Fig. 3), which is an indication that the relaxing phase is dominated by the ring-chain equilibrium and weakly affected by the growing presence of As owing to the nearly constant behavior of $m$. However, in contrast to elemental sulfur for which a sharp change of viscosity and relaxation time is observed [8] and results from $S_{8}$ ring opening and polymerization to ultralong chains having a sluggish diffusional motion (chain entanglements, etc.), the alloying of As leads to a much less sharp transition [18] while also reducing the value of $T_{\lambda}$ (Fig. 3). On the other hand, for $10 \%$ liquids 
one has $T_{g}^{(1)} \leqslant T_{g}^{(2)} \leqslant T_{\lambda}$, which might be an indication that phase II is indeed a mixture of $S_{n}$ chains and rings.

Our data clearly indicate that such complex two-channel relaxation processes $\tau_{\alpha}^{1}$ and $\tau_{\alpha}^{2}$ vanish at around 30\% As as also acknowledged from the corresponding $g_{2}(t)$ function (not shown). An inspection of Figs. 2 and 3 suggests that the $T_{\lambda}$ transition merges into the As-S network glassy dynamics for reversibility window compositions $(\simeq 25 \%)$ [7]. This is also caused by the fact that glasses with arsenic content as high as $20-25 \%$ have already a very high viscosity which will not induce a major change in viscosity if a few more $\mathrm{S}_{8}$ rings open and polymerize into the already existing network, in sharp contrast with elemental sulfur [8]. This is especially true since in this high As regime, there is a negligible fraction of $S_{8}$ rings and the possibility to form long $S_{n}$ chains is practically zero.

Since these results connect to reversibility windows [13] and intermediate phases $[19,20]$, we use them to scrutinize topological constraint theory (TCT) [9], which uses an atomic constraint density $n_{c}$ to predict bulk properties of glasses and supercooled liquid systems such as Vickers hardness [21] or fragility [22]. In most chalcogenides such as the present As-S system, a hierarchy of three types of constraints is considered: $\alpha$ constraints representing linear bonds that form between species, $\beta$ constraints which are angular constraints centered on the As atoms, and $\gamma$ constraints that are sulfurrelated angular interactions which maintain the S-centered angle fixed at its average value. We restrict ourselves to a mean-field approach which permits determining a single isostatically constrained composition $\left(n_{c}=3\right)$, the difference being to infer the effect of phase separation on the prediction of TCT. Since both topological degrees of freedom $f=3-n_{c}$ and configurational entropy $S_{c}$ are extensive functions, using the Adam-Gibbs functional for the relaxation time and the topological estimate of $S_{c}=S_{c}^{(1)}+S_{c}^{(2)} \propto f$ [23] and building on the construction of Ref. [9], we can write the evolution of $T_{g}(x)$ for $x \leqslant 25 \%$ as a function of a glass transition temperature $T_{g}\left(x_{R}\right)$ at a reference composition $x_{R}$ :

$$
\begin{aligned}
\frac{T_{g}(x)}{T_{g}\left(x_{R}\right)} & =\frac{S_{c}\left(x_{R}\right)}{S_{c}(x)}=\frac{\xi\left[3-n_{c}^{(2)}\left(x_{R}\right)\right]+(1-\xi)\left[3-n_{c}^{(1)}\left(x_{R}\right)\right]}{\xi\left[3-n_{c}^{(2)}(x)\right]+(1-\xi)\left[3-n_{c}^{(1)}(x)\right]} \\
& =\frac{1+\frac{\xi-1}{3-2 \xi} n_{c}^{(1)}\left(x_{R}\right)}{1+\frac{\xi-1}{3-2 \xi} n_{c}^{(1)}(x)},
\end{aligned}
$$

where $\xi$ is the fraction of $\mathrm{S}$ atoms part of phase II with relaxation time $\ln \tau_{\alpha}^{2} \propto 1 / T S_{c}^{(2)}$, decoupled from the phase I As-S backbone structure with $\ln \tau_{\alpha}^{1} \propto 1 / T S_{c}^{(1)}$. Here $n_{c}^{(1)}$ and $n_{c}^{(2)}$ represent the constraint densities of phases I and II, respectively. In Eq. (3) a reference composition $x_{R}$ is used and by virtue of the nature of the pure S-phase II, one has $n_{c}^{(2)}=1$ whatever the considered composition since the network coordination number is always 2 , and one also has $\xi\left(x_{R}\right)=1$ if $x_{R}=0$ is chosen. For phase I, following exactly the same strategy as for a similar chalcogenide (Ge-Se [9]), one has $n_{c}^{(1)}=3 x$ using the rules and constraint hierarchy of TCT, i.e., it is assumed that $\alpha$ constraints are frozen in just below the lowest glass transition temperature in composition space $\left[T_{g}(0)\right]$. Equation (3) then leads to the final result, which is given by $T_{g}(0) / T_{g}(x)=1-\beta x$, where $\beta$ is the GibbsDi Marzio constant [24] $\beta=3(1-\xi) /(3-2 \xi)$. The result of the application of TCT for phase I $(\xi=0)$ is given in Fig. 3 as a solid line and predicts with excellent accuracy the overall trend of $T_{g}$ and $T_{g}^{(1)}$ with composition, except for compositions belonging to the intermediate phase [1], where it is known that a mean-field treatment of rigidity does not apply $[16,25,26]$.

In conclusion, by taking advantage of IRPCS measurements, we have been able to rationalize the unusual relaxation behavior of archetypal chalcogenides by measuring their density autocorrelation function $g_{2}$, providing evidence of two typical timescales that are linked to the presence of a nanoscale phase separation. The origin and the behavior with temperature and composition seem to be linked to (i) the reminiscent presence of the $T_{\lambda}$ transition of elemental sulfur, (ii) obvious aspects of structure with a continuous growth of the network connectivity, and (iii) the evolution of the density of topological constraints arising from the constrained atomic interactions. The relaxation channels explored in this study might be related to the fact that sulfur-rich binary As-S glasses exhibit the most prominent photostructural effects, observed in chalcogenides, as a result of the rich variety of species at the nanoscale, including rings, chains, and networklike structures [27]. It would certainly be interesting to probe other sulfur-based supercooled liquids (Ge-S and As-Ge-S) in order to check for the generality of such findings.

T.S. acknowledges support from the PRIN 2017 Project 201795 SBA3 HARVEST and Ateneo Ricerca 2017.
[1] M. Micoulaut, Rep. Prog. Phys. 79, 066504 (2016).

[2] F. Kargl, A. Meyer, M. M. Koza, and H. Schober, Phys. Rev. B 74, 014304 (2006).

[3] B. Ruta, Y. Chushkin, G. Monaco, L. Cipelletti, E. Pineda, P. Bruna, V. M. Giordano, and M. Gonzalez-Silveira, Phys. Rev. Lett. 109, 165701 (2012).

[4] B. Ruta, F. Zontone, Y. Chushkin, G. Baldi, G. Pintori, G. Monaco, B. Rufflé, and W. Kob, Sci. Rep. 7, 3962 (2017).

[5] R. Böhmer, K. L. Ngai, C. A. Angell, and D. J. Plazek, J. Chem. Phys. 99, 4201 (1993).
[6] T. Scopigno, D. Cangialosi, and G. Ruocco, Phys. Rev. B 81, 100202(R) (2010).

[7] P. Chen, C. Holbrook, P. Boolchand, D. G. Georgiev, K. A. Jackson, and M. Micoulaut, Phys. Rev. B 78, 224208 (2008).

[8] T. Scopigno, S. N. Yannopoulos, F. Scarponi, K. S. Andrikopoulos, D. Fioretto, and G. Ruocco, Phys. Rev. Lett. 99, 025701 (2007).

[9] P. K. Gupta and J. C. Mauro, J. Chem. Phys. 130, 094503 (2009). 
[10] S. R. Williams and W. van Megen, Phys. Rev. E 64, 041502 (2001).

[11] M. Bhatt and A. Jamieson, Macromolecules 22, 2724 (1989).

[12] S. N. Yannopoulos, in Amorphous Chalcogenides: Structure, Properties, Modeling and Applications, edited by A. V. Kolobov and K. Shimakawa (World Scientific, Singapore, in press), p. 251.

[13] S. Chakravarty, R. Chbeir, P. Chen, M. Micoulaut, and P. Boolchand, Front. Mater. 6, 166 (2019).

[14] S. V. Nemilov, Sov. Phys. Solid State 6, 1075 (1964).

[15] J. C. Mauro, Y. Yue, A. J. Ellison, P. K. Gupta, and D. C. Allan, Proc. Natl. Acad. Sci. USA 106, 19780 (2009).

[16] M. Micoulaut, Phys. Rev. B 74, 184208 (2006).

[17] T. Wagner, S. O. Kasap, M. Vlček, M. Sklenárí, and A. Stronski, J. Non-Cryst. Solids 227-230, 752 (1998).
[18] K. S. Andrikopoulos, A. G. Kalampounias, and S. N. Yannopoulos, Phys. Rev. B 72, 014203 (2005).

[19] Q. Zhou, M. Wang, L. Guo, P. Boolchand, and M. Bauchy, Front. Mater. 6, 157 (2019).

[20] L. Yan, Nat. Commun. 9, 1359 (2018).

[21] M. M. Smedskjaer, J. C. Mauro, and Y. Yue, Phys. Rev. Lett. 105, 115503 (2010).

[22] K. H. Lee, Q. Zheng, J. Ren, C. J. Wilkinson, Y. Yang, K. Doss, and J. C. Mauro, J. Non-Cryst. Solids 521, 119534 (2019).

[23] G. G. Naumis, Phys. Rev. E 71, 026114 (2005).

[24] A. N. Sreeram, D. Swiler, and A. K. Varshneya, J. Non-Cryst. Solids 127, 287 (1991).

[25] M.-A. Brière, M. V. Chubynsky, and N. Mousseau, Phys. Rev. E 75, 056108 (2007).

[26] H. Kriegs, J. Chem. Phys. 124, 104901 (2006).

[27] F. Kyriazis and S. N. Yannopoulos, Appl. Phys. Lett. 94, 101901 (2009). 